\title{
A Translational Analysis of Renewal Following DRO with Human Subjects
}

Kacey R. Finch

West Virginia University, krf0009@mix.wvu.edu

Follow this and additional works at: https://researchrepository.wvu.edu/etd

Part of the Experimental Analysis of Behavior Commons

\section{Recommended Citation}

Finch, Kacey R., "A Translational Analysis of Renewal Following DRO with Human Subjects" (2021). Graduate Theses, Dissertations, and Problem Reports. 8133.

https://researchrepository.wvu.edu/etd/8133

This Thesis is protected by copyright and/or related rights. It has been brought to you by the The Research Repository @ WVU with permission from the rights-holder(s). You are free to use this Thesis in any way that is permitted by the copyright and related rights legislation that applies to your use. For other uses you must obtain permission from the rights-holder(s) directly, unless additional rights are indicated by a Creative Commons license in the record and/ or on the work itself. This Thesis has been accepted for inclusion in WVU Graduate Theses, Dissertations, and Problem Reports collection by an authorized administrator of The Research Repository @ WVU. For more information, please contact researchrepository@mail.wvu.edu. 
A Translational Analysis of Renewal Following DRO with Human Subjects

\author{
Kacey R. Finch
}

Thesis submitted to the Eberly College of Arts and Sciences at West Virginia University in partial fulfillment of the requirements for the degree of Master of Science in Psychology

\author{
Kathryn Kestner, Ph.D., BCBA-D, Chair \\ Claire St. Peter, Ph.D., BCBA-D \\ Barry Edelstein, Ph.D. \\ Department of Psychology
}

\author{
Morgantown, West Virginia \\ 2021
}

Keywords: Differential reinforcement, DRO, human operant, relapse, renewal

Copyright 2021 Kacey R. Finch 


\begin{abstract}
A Translational Analysis of Renewal Following DRO with Human Subjects

Kacey R. Finch

Renewal is a type of behavioral relapse that is dependent on changes in contextual stimuli in the environment. The current study investigated ABA renewal of responding that had been previously reduced by a differential reinforcement of other behavior (DRO) procedure. ABA renewal refers to the context associated with three phases of behavioral treatment; Context $\mathrm{A}$ is present during baseline, Context $\mathrm{B}$ is present during treatment, and the post-treatment context returns to Context A (i.e., renewal test). Undergraduate college students participated in a Zoom video call during which they responded by clicking the mouse on a rectangle in a computer program created in Visual Basic ( $)$. Each session consisted of baseline, DRO, and a renewal test phase. In baseline, the target response was reinforced according to a fixed-interval (FI) 10-s schedule. In the DRO phase, points were delivered contingent on refraining from clicking on the target response during the 10 -s omission interval. The same DRO contingency was in effect during the renewal test. A change in the color of the screen background served as the contextual change between baseline, treatment, and renewal test phases. This study assessed the occurrence of renewal via visual inspection of graphed data. Renewal occurred for all six included participants and was transient for five of six participants. Proportion of baseline and reinforcement rate data are presented in addition to target and untargeted response rate. Implications for clinical application and future research directions are discussed.
\end{abstract}




\section{Table of Contents}

Introduction.............................................................................1

Method.......................................................................................... 7

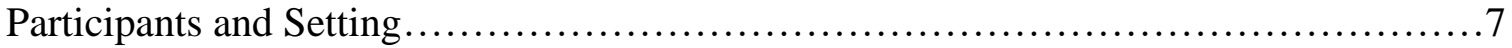

Apparatus............................................................... 8

Procedure, Experimental Design, and Dependent Measures.......................... 8

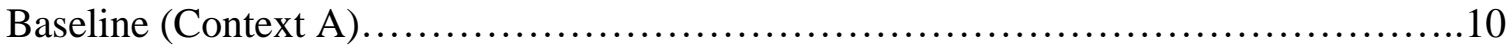

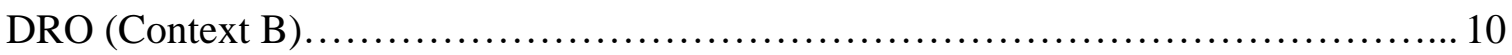

Renewal Test (Context A).................................................... 10

Data Analysis...................................................................... 11

Results................................................................................ 12

Discussion................................................................................. 17

Future Directions..................................................... 20

References..............................................................................26

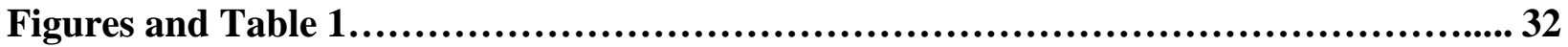

Appendix A................................................................................. 38

Appendix B.......................................................................... 40 


\section{A Translational Analysis of Renewal Following DRO with Human Subjects}

Relapse is said to occur when a previously reduced response reemerges following treatment. Relapse is common following the cessation of, or a challenge to, treatment procedures and is often countertherapeutic (Wacker et al., 2011). For example, relapse of challenging behavior (e.g., aggression) can be dangerous and can compete with more desirable alternative responses (e.g., verbal requests for access to preferred items). Applied behavior analysts, therefore, have a direct interest in promoting the persistence of treatment effects on socially significant behavior and prevention of relapse of undesirable behavior across time. Two of the most commonly studied types of relapse include resurgence (i.e., relapse of a response after reinforcement conditions are worsened for an alternative response; Lattal et al., 2017) and renewal (i.e., relapse of responding when the treatment conditions are carried out under different conditions post-treatment; see Bouton \& Todd, 2014 for a review). Resurgence and renewal have been studied across basic literature with non-human animals and applied literature with human subjects.

Kelley and colleagues (2015) define a context change as any stimulus change from the environment that previously controlled behavior (e.g., changes in lights or olfactory stimuli in an operant chamber, changes in the background color in a computer program, or changes in physical location such as moving from a clinic to home). Renewal has been demonstrated in both human (e.g., Ibanez et al., 2019; Liddon et al., 2018; Saini et al., 2018) and non-human animal populations (e.g., Craig et al., 2018; Kelley et al., 2015). Renewal is typically evaluated in a three-phase sequence that varies contextual stimuli across each phase (e.g., Liddon et al., 2018). A recent study on the prevalence of clinical renewal assessed 182 context changes across 67 clients receiving outpatient applied behavior analysis services (Muething et al., 2020). Renewal 
occurred in 77 of 182 context changes (i.e., a $42.3 \%$ renewal occurrence rate). There are several types of renewal based on the pattern of changes in contextual stimuli. In ABA renewal, the target response (e.g., challenging behavior) is reinforced in Context A. In Context B, a responsesuppression procedure (e.g., extinction) is in effect for the target response. In the third phase, Context $\mathrm{A}$ is re-introduced, and the response-suppression procedure remains in effect for the target response. In ABC renewal, a third context is introduced in the third phase in which the deceleration procedure is still in effect for the target response. Renewal is assessed based on whether there is a recurrence of the target response in the third phase when the only change in the environment is the context change.

Renewal of a previously reduced response is most commonly studied with extinction (e.g., Bouton et al., 2011; Bouton \& Ricker, 1994) or differential reinforcement of alternative behavior (DRA) as the treatment method. In a series of experiments assessing ABA renewal, Kelley and colleagues (2015) conducted a basic laboratory analysis with pigeons followed by a translational analysis of ABA renewal with children with autism. The procedure for each experiment was similar such that reinforcement was implemented for key pecking for pigeons in baseline (Context A) of Experiment 1 and for on-task behavior for participants in Experiment 2. In the treatment phase (Context B), extinction was in effect for key pecking and on-task behavior, respectively. In the renewal test, the context was the same as baseline (i.e., Context A) and extinction was still in effect. Renewal occurred for all six pigeons in Experiment 1 and both participants in Experiment 2. This series of experiments demonstrated that renewal occurred in similar patterns across basic and clinical settings in both populations. Notably, Experiment 2 showed renewal of a desirable response after it was extinguished in the second phase. In addition to analyzing renewal of previously extinguished appropriate behavior, renewal of challenging 
behavior that is reduced in treatment is another important phenomenon to study. Renewal of challenging behavior is often a barrier to generalization of treatment effects (e.g., alternative behavior increased in treatment) and maintenance of behavior change. Identifying interventions that are more resistant to relapse or methods to decrease the likelihood of relapse is crucial to promoting maintenance and generalization of therapeutic effects (e.g., treatment-correlated stimuli, multiple-context training; see Wathen \& Podlesnik, 2018 for a review).

In a clinical example of renewal, a client may have a history of receiving attention from parents following instances of aggression in the naturalistic environment (i.e., home). When the client is brought to the treatment context (e.g., clinic) where they receive intensive applied behavior analysis services, a DRA procedure may be implemented in which attention is provided contingent on appropriate behavior and extinction is programmed for aggression (i.e., the adults do not provide attention contingent on aggression). Following a successful decrease in aggression at the clinic, returning to the original context in which the behavior was previously reinforced (i.e., home) may result in renewal of aggression, even if the parents continue the same intervention at home. Although DRA and extinction are common treatment components in renewal analyses, another behavior-reduction technique implemented in clinical settings is differential reinforcement of other behavior (DRO), which decreases a target response by providing reinforcement contingent on the absence of the response (Homer \& Peterson, 1980). For example, if the goal is to reduce aggression, a DRO procedure might involve providing access to attention contingent on the client refraining from engaging in aggression for the duration of a predetermined interval (e.g., 2 min). Engaging in the response during the interval is not reinforced, which is a key component of the response-suppressive effect of DRO (i.e., extinction, see Jessel \& Ingvarsson, 2016 for a review of active mechanisms of DRO). While 
differential reinforcement procedures, such as DRO, are common and effective interventions for decreasing challenging behavior, a response decreased via differential reinforcement is prone to relapse following treatment (Lattal \& St. Peter Pipkin, 2009). Challenging behavior has been shown to relapse following differential reinforcement (e.g., functional communication training) when it is implemented in different contexts or by different people, even with high treatment integrity (e.g., caregivers implementing treatment at home following a reduction of the response in a clinical setting; Schindler \& Horner, 2005). This context-dependent relapse is an example of renewal because contextual stimuli change from the original treatment environment to posttreatment environments. One hypothesis to explain the occurrence of renewal is that the effects of extinction might be specific to the context in which the treatment was initially implemented (Bouten \& Todd, 2014).

Renewal is increasingly being studied in both translational and applied settings to learn more about the role of contextual changes in the occurrence of relapse. Translational research assessing renewal in human subjects has significantly increased in the last two years (e.g., Ibańez et al., 2019; Kimball et al., 2020; Liddon et al., 2018; Novak et al., 2020; Robinson \& Kelley, 2020; Schlund et al., 2020). Much of the existing literature on renewal assesses responding following either an extinction or DRA treatment. Although DRO is a treatment strategy implemented in clinical settings, less research has specifically investigated the prevalence of renewal following a DRO procedure, and most of these DRO studies have been with non-human animals as subjects.

Three studies have investigated renewal in rats as subjects following a DRO schedule in the second phase of the renewal paradigm (Kearns \& Weiss, 2007; Nakajima et al., 2002; Rey et al., 2020b). Nakajima et al. (2002) compared responding between an ABA renewal test group 
and an AAA control group. In Phase 1, responding on the target lever was reinforced. The DRO contingency was implemented in Context B for the ABA group and in Context A for the AAA group. In the renewal test phase, DRO remained in effect for the target response such that the only difference between the treatment phase and the renewal test phase for the ABA group was the context. Renewal occurred in the ABA renewal test group and not in the AAA control group, which provides support that renewal can be attributed to the context change in the ABA renewal group from Phase 2 to Phase 3.

The remaining two non-human animal studies that assessed renewal following DRO discontinued the DRO schedule in the renewal test and extinction was in effect for all responses (Kearns \& Weiss, 2007; Rey et al., 2020b). Using an ABA procedure, Kearns and Weiss compared renewal following DRO and extinction in the second phase (Context B). The results demonstrated that the level of renewal of responding eliminated by a DRO procedure was significantly lower than the level of renewal following extinction. This suggests that behavior reduced using a DRO schedule may be more resistant to relapse compared to extinction. In another analysis, Rey et al. compared renewal following DRO and extinction in two groups of rats. Renewal occurred for 15 out of 16 subjects in the DRO group and all 16 subjects in the extinction group; the levels of renewal between groups were not directly compared in this study. The results of these three studies demonstrate that renewal is common following DRO. Although the results from Kearns and Weiss suggest that there may be differences in the levels of renewal following different response-suppression procedures, additional research is needed to determine whether such differences consistently occur.

Only one known experiment has assessed renewal following a DRO procedure with human subjects (Vila et al., 2020). In this study, experimenters arranged a video game in which 
participants were asked to shoot at virtual spaceship enemies. Two responses were differentially consequated across the three phases of a trial-based renewal arrangement. In Phase 1, both responses were reinforced according to a variable-interval (VI) 4-s schedule in eight trials, and all trials ended after a single reinforcer delivery. In Phase 2, a DRO schedule was in effect for one response with a 4-s omission interval for the next eight trials. Participants in the ABA group experienced Phase 2 in a different context than Phase 1; the context change included a different colored computer and keyboard and different colored furniture in the experimental room. The context did not change from Phase 1 to Phase 2 for the AAA group. The renewal test phase occurred in Context A and consisted of one trial in which the DRO was discontinued and no reinforcers were delivered for responding. The response associated with the DRO schedule increased from Phase 2 to the Phase 3 test trial in the ABA group but did not increase for the AAA control group, suggesting that renewal occurs in human subjects following response reduction by a DRO procedure. However, it is unclear whether the context change alone is responsible for the occurrence of renewal because the reinforcement schedule and the context changed from Phase 2 to Phase 3. Another limitation of this study is that testing renewal in a single trial precludes an evaluation of the persistence and pattern of relapse following a sustained change in context.

Most renewal arrangements implement extinction in the treatment phase and then continue the implementation of extinction in the renewal test phase (Saini \& Mitteer, 2020). Of the four studies on renewal following DRO, three discontinued DRO and implemented extinction of all available responses in the renewal test phase (Kearns \& Weiss, 2007; Rey et al., 2020b; Vila et al., 2020). This arrangement introduces a confound of two variable changes from the treatment phase to the renewal test phase (the context and the reinforcement schedule). One 
study, however, demonstrated the occurrence of renewal when the same DRO contingency continued from the treatment phase during the renewal test phase (Nakajima et al., 2002). This arrangement isolated the context stimulus as the only variable that changed from the treatment phase to the renewal phase and mirrors the common clinical occurrence that the intervention remains in effect post-treatment (e.g., the parents may be trained to implement the DRO schedule at home).

The purpose of this experiment was to further assess the occurrence of ABA renewal following a DRO procedure (rather than the more common DRA or extinction procedures) to reduce a target response in a highly controlled human-operant arrangement. The current experiment extended previous research by arranging a renewal test in which the DRO contingency from the treatment phase remained in effect during the renewal test phase (e.g., Nakajima et al., 2002) and extended the duration of the renewal test beyond the single-trial test by Vila et al. (2020). We conducted the current experiment via Zoom to assess the utility of virtual platforms for efficiently gathering behavioral data on relapse (e.g., Robinson \& Kelley, 2020).

\section{Method}

\section{Participants and Setting}

Ten undergraduate students (six female and four male) from WVU ages 18-22 participated in virtual sessions and received course credit in a psychology course. Nine participants reported their race as White, and one participant reported their race as Black. Requirements to participate included access to a computer equipped with a mouse or trackpad, speakers or headphones, a webcam, a microphone, an internet connection, and Zoom for video calling. We conducted all 
sessions through Zoom, and the participants attended the sessions from their home or other personal work area using a personal computer. The experimental task was screen shared with the participant via Zoom, and the participant was given remote control of the screen such that they could interact with the computer program using their mouse and keyboard.

\section{Apparatus}

The experimental task was programmed in Microsoft Visual Basic $\subset$ and included one $6.0-\mathrm{cm}$ by $1.3-\mathrm{cm}$ stationary black rectangle in the middle of the screen. The participant clicked on a white start button at the beginning of the session to start the program. Following a mouse click on the black rectangle (i.e., target response), the rectangle turned gray for $100 \mathrm{~ms}$. When the participant met the criterion for earning a point according to the fixed-interval (FI) $10-\mathrm{s}$ schedule or the DRO 10-s schedule, the rectangle temporarily disappeared, a box appeared that said " +1 " and the participant clicked a button below the text that said, "collect point" (i.e., consummatory response). During the consummatory response, the omission interval in the DRO phase and the interval in the FI schedule were paused. Clicks on the background area of the screen resulted in no programmed consequences across all three phases. There was a cumulative point counter present throughout the session at the top of the screen, and a point was added following the consummatory response. After the point was delivered, the rectangle reappeared in the middle of the screen. Points were not exchanged for backup reinforcers. The background colors of the screen (yellow and blue) served as the contextual stimuli and changed from phase to phase.

\section{Procedure, Experimental Design, and Dependent Measures}


Each participant attended one virtual 60-min session with exposure to all three phases of the renewal arrangement. Each phase was $15 \mathrm{~min}$, and the remainder of the 60-min appointment included informed consent at the beginning and a demographic questionnaire at the end. We explained the informed consent form to the participant at the beginning of the Zoom session, and the participant provided consent by typing their name in a box on Qualtrics. During the informed consent process, we described expectations for the participant (e.g., keep pets away from computer screen, limit distractions as much as possible, keep video on). Following informed consent, we read the participant the following instructions: "The experiment involves a computer task. There will be a point counter at the top of the screen. It is up to you to figure out how to earn points. Do you have any questions?" The experimenter did not reveal any of the contingencies of the experiment (e.g., the experimenter did not answer questions such as "why am I not earning points anymore?"). The experimental design was a 3-phase ABC design that included a baseline phase (Context A) in which the target response was reinforced, a DRO treatment phase (Context B) to reduce the target response, and a renewal test phase (return to Context A). The primary dependent measure was the frequency of mouse clicks on the target response, which was automatically recorded and timestamped by the program and is reported as the number of responses per min in each 15-min phase. The experimenter running the session was available on Zoom for any questions or technology concerns but was on mute and had their video off while the participant was engaged in the experimental task. Phases were associated with different stimulus contexts, represented by different background colors of the screen. Specifically, the color was yellow in Phases 1 and 3 (i.e., Context A) and blue in Phase 2 (i.e., Context B). At the end of the session, participants completed a demographic questionnaire (Appendix A) via a Qualtrics survey. The questionnaire also included three open-ended 
questions that asked the participants to explain what they guessed was the purpose of the experiment. We then read the following debriefing statement to the participant: "Thanks for participating in this research. The purpose of this study was to look at patterns of behavior when the ways to earn points change across the different screen colors. This computer program is set up to be an analog of a clinical treatment that is commonly used in behavior analysis to reduce undesirable behavior. The screen colors represent different physical locations that a client might be in over time (e.g., a clinic, a school, home). We hope this study will inform clinical interventions that have better long-term effects on behavior change. We are continuing to recruit participants, so please do not share details about this session or the purpose of this project with anyone. Do you have any questions?"

\section{Baseline (Context A)}

The background color during baseline was yellow. Clicking on the rectangle was reinforced according to an FI-10 s schedule in which the first mouse click on the rectangle after the 10-s interval elapsed resulted in the delivery of one point.

\section{DRO (Context B)}

In the DRO phase, one point was delivered following the omission of responding on the target for the entirety of the 10-s omission interval. Each time mouse clicks on the target response occurred, the DRO interval was reset, and the scheduled reinforcer was canceled. The background color during the DRO phase was blue.

\section{Renewal Test (Context A)}

In the renewal test, the contingencies for the target response were the same as during the DRO phase. To isolate the change in context, the only difference between the DRO phase and 
the renewal test was the background color, which returned to the same color as baseline (i.e., yellow).

\section{Data Analysis}

We analyzed the results via visual inspection of graphed data to compare responding in the treatment and renewal test phases (see Figure 1 and 2). Renewal was defined as the number of mouse clicks on the rectangle in any given minute of the renewal test exceeding the average responses per min from the last 5 min of the DRO phase. We also calculated the proportion of baseline responding in the renewal test for each participant by dividing the number of responses in each minute of the 15-min renewal test by the mean number of responses from the last $5 \mathrm{~min}$ of baseline (see Figures 3 and 4). The purpose of calculating the proportion of baseline was to provide a relative measure of renewal compared to each individual participant's baseline response rate. The number of reinforcers delivered in each min was also recorded by the computer program (see Figure 5 for included datasets and Figure 6 for excluded datasets). Untargeted behavior was defined as mouse clicks on the background screen and was visually analyzed for all included datasets (see Figure 7). Consummatory response time was defined as the time from the presentation of the consummatory message on the screen (i.e., the "collect point" box) to the participant clicking on the "collect point" message and the box disappearing. Consummatory response time was analyzed to determine if there were differences between time spent with the consummatory message across phases (i.e., if consummatory response time was greater in the DRO phase compared to baseline) because increased consummatory response time across the session may indicate participant fatigue. A participant's data were excluded from data analysis if the participant engaged in less than 10 responses per min or a downward trend in graphed data in the last $5 \mathrm{~min}$, as this would suggest that points do not function as reinforcers for 
that participant. Data were also excluded from data analysis if responding was not suppressed in the DRO treatment phase.

\section{Results}

Ten participants completed the study; six data sets were included, and datasets from four participants were excluded from the analysis based on the exclusion criteria. Participant 302 was excluded because responding was not suppressed in the DRO treatment phase, Participant 304 was excluded because responses per min were trending downward in the last 5 min of baseline, and Participants 305 and 309 were excluded because the average responses per min in the last 5 min of baseline was less than 10 (see Figure 1 for all excluded participant target response data and Appendix B for supplemental graphs for these datasets). Renewal of target responding occurred for all six included participants (see Figure 2). All included participants acquired the target response in the baseline phase, and the mean number of responses per min in the last 5 min of baseline ranged from 33.2-176.8. In the DRO treatment phase, responding decreased from baseline levels to near-zero levels for all included participants. Mean responses per min in the last 5 min of the DRO treatment phase ranged from $0-0.442$. The proportion of baseline data provide values that measure the occurrence of renewal relative to each participant's baseline response rate as baseline responding differed across participants. The proportion of baseline during the minutes in which renewal occurred ranged from $0.27-0.86$, indicating that the intensity of renewal varied across participants as measured by a comparison to baseline responding. The mean number of reinforcers delivered per minute ranged from $4-4.87$ in baseline, 2.8-4.87 during the DRO treatment phase, and 4.27-5.27 during the renewal test phase for included datasets (see Figure 3). The number of reinforcers delivered per min was similar across included datasets. In general, when phases changed from baseline to DRO treatment or 
from DRO treatment to the renewal test, obtained reinforcer delivery decreased temporarily but stabilized to levels similar to baseline as target responding stabilized. There was a negative relation between the number of reinforcers delivered and level of renewal across minutes (i.e., when target responding was high in the renewal test, the number of reinforcers was low). Untargeted behavior (i.e., clicks on the background screen) varied across participants and is reported below for each participant. Total consummatory response time did not vary systematically across phases or participants and ranged from 3.72-7.16 $\min (1.20-2.51 \mathrm{~min}$ for Phase 1; .56-2.19 min for Phase 2; 1.13-2.73 min for Phase 3; see Table 1).

In the baseline phase for Participant 301, mouse clicks on the target rectangle were elevated but variable (i.e., rate changed across the phase but lacked an upward or downward trend), with the highest number of responses per min occurring in the last 5 min of baseline at an average of 58.8 responses per min (see Figure 2). Responding during the first min of the DRO phase (i.e., 164 responses) was higher than any min of the baseline phase (maximum responding in the baseline phase was 134 clicks in min 12) and decreased to zero responses per min by the fourth min of the DRO phase and remained under two clicks per min for the rest of the phase. Mouse clicks occurred an average of 0.44 times per min in the last five min of the DRO treatment phase. The number of responses per min in the renewal test ranged from 0-37 and proportion of baseline ranged from $0-0.63$. Renewal occurred in the first min of the renewal test (proportion of baseline $=0.63$; see Figure 4 ), then responding reduced to zero or near-zero levels for the remainder of the phase. During baseline, untargeted responding was variable (range, 075) and occurred a mean of 0.6 times per min during the last 5 min of the phase (see Figure 5). Untargeted behavior (i.e., background clicks) occurred 10-24 times per min during the first 3 min of the phase and then decreased to near-zero rates for the remainder of the phase, with no 
occurrences in the last 3 min of the phase. During the first min of the renewal test, untargeted behavior initially increased to 9 clicks in the first min and then decreased to near-zero levels. Untargeted behavior generally increased when renewal occurred.

For Participant 303, target responding was elevated above zero for the entirety of the baseline phase, and Participant 303 engaged in an average of 74.6 responses per min in the last five min of baseline (see Figure 2). Responses per min trended downward in min 10-13 but increased to the highest rate of responding for the entire session in minutes 14 (157 responses) and 15 (138 responses). At the beginning of the DRO treatment phase, responding was elevated at an average of 75.4 responses per min for the first five min. Responding decreased to zero responses per min by min 8 and remained at zero for the rest of the phase; therefore, average responses per min in the last 5 min of the DRO phase was zero. In the first min of the renewal test phase, one target response occurred, then responding returned to zero for the remainder of the session. The maximum proportion of baseline was 0.01 for Participant 303 (see Figure 4). Responding in the renewal test exceeded responding from the end of the treatment phase; therefore, renewal did occur. The pattern of untargeted behavior was similar to the pattern of target behavior across all three phases, although the level of target responding was generally higher than untargeted responding (see Figure 5). Background clicks occurred variably in baseline and ranged from 0-18 times per min. Untargeted behavior occurred between 6 and 30 times in the first 6 min of the DRO treatment phase and then decreased to zero for the last $7 \mathrm{~min}$ of the phase. Untargeted behavior did not occur in the renewal test phase.

For Participant 306, target responding was variable but lacked an upward or downward trend in baseline, with responding ranging from 10 to 50 responses per min (see Figure 2). Target responding occurred an average of 33.2 times per min in the last 5 min of baseline. Responding 
in the first three min of the DRO treatment phase was similar to baseline responding, ranging from 34-44 and then increased to 84 responses in the fourth min. Responding then trended downward and suppressed to zero by the eighth min of the DRO treatment phase. Mean responses per min was zero for the last 5 min of the DRO treatment. Renewal occurred in the first min of the renewal test phase with nine clicks on the target rectangle. The maximum proportion of baseline was 0.27 (see Figure 4). Untargeted behavior occurred at a low rate throughout the baseline phase (range 1-16) and the first 6 min of the DRO treatment phase (range 0-4; see Figure 5). Then untargeted behavior increased and remained elevated for the remainder of the session. The mean number of responses per min was 80 responses per min for the last 5 min of the DRO treatment phase and 82.2 for the entire renewal test phase.

Participant 307 engaged in a mean of 141.2 target responses per min in the last five min of baseline (see Figure 2) and trended upward during the last $10 \mathrm{~min}$ the phase. In the DRO treatment phase, 145 and 57 responses occurred in the first and second min, respectively. Responding then decreased to zero in the third min and remained at zero for the rest of the phase. Participant 307 clicked 122 times on the target rectangle in the first min of the renewal test phase; therefore, renewal did occur. Responding did not occur for the rest of the test phase. The proportion of baseline for the first min of the renewal test was 0.86 (see Figure 4). Untargeted behavior was elevated in the first four min of the baseline phase (range, 15-33) before decreasing to near-zero levels (see Figure 5). Untargeted behavior occurred in the first 3 min of the DRO treatment phase 14 times, 50 times, and 29 times, respectively. Untargeted behavior did not occur for the remainder of the DRO treatment phase. Background clicks occurred 4 times in the first min of the renewal test and 1 time in the fourth min of the renewal test but did not occur in any other min of the renewal test. 
For Participant 308, target responding in baseline was variable but elevated for the entirety of the phase (range 66-203). Participant 308 clicked on the target rectangle a mean of 172.8 times per min in the last five min of baseline (see Figure 2). Responding in the first five min of the DRO treatment phase trended downward during the first 3 min, reached zero by the third min, and stayed at zero for the remainder of the phase. The average responses per min was zero in the last five min of the DRO treatment phase. The responses per min in the renewal test ranged from $0-115$ and proportion of baseline ranged from $0-0.67$ (see Figure 4). In the first min of the renewal test, Participant 308 responded 115 times on the target rectangle and then decreased to zero responses for min 3 and min 4 . Responding was variable for the rest of the phase, ranging from zero to 32 responses per min. Mouse clicks on the target rectangle increased to six in min 39 and 32 clicks in min 43. Therefore, renewal occurred for Participant 308, and responding persisted at lower levels throughout the renewal test phase. Untargeted behavior occurred sporadically at low levels throughout the baseline phase (range 0-12), then increased in the first 4 min of the DRO treatment phase (range 14-102) before decreasing and stabilizing at 0 responses per min at the end of the phase (see Figure 5). In the renewal test phase, untargeted behavior increased at the beginning of the phase and occurred 17 times in the first min and 39 times in the second min. Similar to target responding in the renewal test, untargeted behavior occurred at elevated levels across the renewal test phase (e.g., 44 times in min 39; 18 times in min 43). When renewal occurred for Participant 308, untargeted behavior generally increased in the same min, suggesting that there was a positive relation between renewal of target responding and occurrence of untargeted behavior for this participant.

For Participant 310, target responding was lowest in the beginning of the baseline phase and increased rapidly to over 200 responses by the third min (see Figure 2). In the last five min 
of baseline, an average of 176.8 responses occurred per min. In the DRO treatment phase, responding was above 200 responses for the first min and quickly decreased to zero responses by the second min. Responding remained low for the rest of the DRO treatment phase and the average responses per min in the last five min was zero. Participant 310 clicked 92 times on the target rectangle in the first minute of the renewal test phase, therefore renewal did occur. Participant 310 did not click on the target rectangle for the rest of the phase. The maximum proportion of baseline was 0.52 in the first min of the renewal test (see Figure 4). Untargeted behavior occurred 23 times in min 1 and 15 times in $\min 2$, then decreased to zero or near-zero levels for the remainder of the baseline phase (see Figure 5). In the DRO treatment phase, untargeted behavior increased temporarily (i.e., 9 times in min 16 and 34 times in min 17), then decreased to near-zero levels for the remainder of the phase. In the renewal test phase, untargeted behavior occurred at low rates at the beginning of the phase but increased variably from min 39 to the end of the phase, with the maximum occurrence at 36 times in min 40 . Untargeted behavior occurred for Participant 310 but did not seem to directly relate to the occurrence of renewal.

\section{Discussion}

The purpose of this study was to assess the occurrence of renewal of target responding following omission training (i.e., DRO). All six participants for whom datasets were included in the analysis acquired the target response in baseline, and responding was suppressed in the second phase during the DRO treatment phase. In general, the data from this study are evidence of renewal following a DRO treatment for all six participants. The proportion of baseline responding for the minutes in which renewal occurred varied across participants, which is indicative of varying levels of renewal relative to individual baseline responding. The results of 
this study are consistent with previous human-operant research that has assessed renewal following omission training according to a DRO schedule (Vila et al., 2020). This study extended Vila et al. by demonstrating renewal when context is isolated as the only variable that changes from treatment to the renewal test, which provides additional evidence that a context change can result in relapse. Additionally, this study demonstrates that systematic humanoperant data can be collected using a virtual approach by providing the participant remote control of a program via Zoom. Orderly data were obtained despite the extraneous variables that may be present in the participant's environment (e.g., pets, roommates, or family members). This study contributes to a modest collection of studies that have employed remote data collection using a free-operant task (e.g., Robinson \& Kelley, 2020).

The data from the current study are consistent with previous research that suggests that renewal is transient such that responding returns to stable treatment levels following renewal (e.g., Muething et al., 2020). Renewal occurred for all six participants and was temporary for five participants. Renewal for Participant 308 was somewhat persistent across the renewal test phase such that responding initially decreased to zero in the third min of the renewal test and then persisted variably throughout the remainder of the phase. Even though renewal was persistent relative to other participants, rate of responding in the renewal test phase was still lower than responding in baseline, which still demonstrates an overall reduction in target responding from baseline to post-treatment conditions. Due to the standardized phase duration at 15 min, it is unknown to what extent target responding would have persisted if the phase would have been longer. The transient nature of renewal has implications for clinical settings and the treatment of challenging behavior. Clinicians are often warned that relapse can occur when reinforcement conditions are worsened (i.e., resurgence, Lattal \& St. Peter Pipkin, 2009), but the 
emerging literature on renewal suggests that clinicians and caregivers should also prepare for relapse following a context change, even when the treatment is still implemented with high integrity. However, with the transient nature of renewal, clinicians and other caregivers can be encouraged that renewal of challenging behavior is likely to be temporary and lower than baseline levels. Practitioners should emphasize that it is important to maintain the treatment contingencies during the increase in challenging behavior.

There are some limitations to this study. First, this experiment did not include a replication of phases (i.e., each phase only occurred once for each participant); demonstrating a replication of renewal following DRO would increase confidence in the interpretation that the DRO schedule decreased target responses and that context changes resulted in renewal. One way to incorporate replication would be to repeat each of these phases with their respective contingencies (i.e., an $\mathrm{ABCABC}$ reversal design). However, drawing parallels from the resurgence literature, less renewal might be expected in a replication via an $\mathrm{ABCABC}$ reversal design (Kestner et al., 2018). A second limitation was the difficulty observing or controlling distractions in the participants' environment with the virtual arrangement (e.g., pets, other people, weather, competing contingencies such as phone usage). Conducting this research in a laboratory setting in person would increase control of potentially confounding variables, which could provide more confidence that responding was not influenced by extraneous variables in the participants' physical environment. However, conducting human-operant research virtually was also a strength of this study as it increased efficiency and decreased cost. Considering the benefits, further analysis of virtual arrangements of human-operant research is a worthwhile area. Future research may assess ways to control for extraneous environmental variables during remote sessions. Third, the extent to which these findings would generalize to clinical settings is 
unknown because many of the conditions arranged in laboratory settings are unlike naturalistic variables (e.g., the target response is an arbitrary analog to challenging behavior). Humanoperant arrangements allow experimenters to analyze relations between independent and dependent variables in a highly controlled setting, but when translated to clinical settings, some extraneous variables can be more difficult to control (e.g., integrity of treatment implementation, responses from peers in classroom settings). However, previous basic laboratory and translational research of relapse have demonstrated continuity with clinical applications. In one example described previously, Nevin et al. (2016) conducted a basic laboratory analysis of resurgence and reinstatement with pigeons in one experiment and a similar procedure with human subjects that engaged in challenging behavior, and the results demonstrated parallel relapse trends across translational and clinical analyses. Translational analyses of behavioral processes are important for bridging the gap between basic research and clinical application (see Critchfield, 2011 for a discussion). The translational nature of the current study extends renewal with DRO that has been previously demonstrated in basic laboratories to human subjects while still maintaining high laboratory control to isolate basic processes.

\section{Future Directions}

Future research should continue to assess the conditions under which renewal occurs following DRO and other behavior reduction intervention strategies. The present study assessed renewal when the renewal test occurred in the same context as baseline (i.e., ABA renewal). There are no published studies to date investigating renewal in an $\mathrm{ABC}$ or $\mathrm{AAB}$ renewal paradigm following a DRO treatment. Studying different renewal paradigms following omission training will be important because context changes that occur in natural environments often mimic arrangements other than $\mathrm{ABA}$ renewal (e.g., $\mathrm{AAB}$ or $\mathrm{ABC}$ renewal). Future research 
should arrange contexts to assess $\mathrm{ABC}$ renewal by measuring target behavior in one context for baseline (e.g., home), a new context for treatment (e.g., clinic), and a third novel context for the renewal test (e.g., school) to better understand how multiple context changes impact the occurrence of renewal. For AAB renewal, the context in baseline and treatment is the same (e.g., home for both phases) and renewal is assessed in a novel, second context (e.g., school). These studies would provide additional information about the occurrence of relapse following DRO given context changes that differ from $\mathrm{ABA}$ renewal but represent patterns of change that likely occur in clinical populations.

Additional research should also compare the occurrence of renewal following various commonly implemented treatments. Future research should compare the prevalence and intensity of renewal following DRA and DRO; identifying interventions that are more resistant to relapse of challenging behavior is crucial to promoting maintenance and generalization of therapeutic effects. Previous translational relapse literature has compared resurgence following a DRO treatment and a DRA treatment using multiple schedules of reinforcement (Romano \& St. Peter, 2017). More resurgence occurred following the DRO intervention relative to the DRA intervention. Similar comparisons could be conducted for renewal, specifically isolating changes in context across phases to better inform which treatments might be more resistant to relapse. Data demonstrating more resurgence and renewal following a DRO treatment might suggest that a DRA treatment is a better option for maintaining treatment effects and decreasing the likelihood of relapse in the form of resurgence and renewal.

In addition to studying relapse with other treatment strategies, further analysis of the treatment effects of DRO procedures is also warranted. Previous research has analyzed the active components of DRO that facilitate decreases in challenging behavior. Mechanisms of behavior 
change in DRO might include extinction by withholding a reinforcer after the occurrence of problem behavior, negative punishment because the delivery of the reinforcer is canceled or postponed contingent on problem behavior, or adventitious reinforcement of untargeted behavior that might occur in close temporal proximity to the delivery of the reinforcer according to the DRO schedule (Jessel \& Ingvarsson, 2016). As the name DRO suggests, untargeted behavior can be defined as any "other" response that occurs during a DRO treatment that is not the target response (Poling \& Ryan, 1982). In a human-operant laboratory setting, examples of untargeted behavior might include clicking on the background of the screen, clicking on the point counter, or pressing keys on the keyboard. In a clinical setting, untargeted behavior could include new or different topographies of challenging behavior not targeted in treatment or the emergence of appropriate behavior that is not specifically reinforced (e.g., task engagement or mands for preferred items). There is evidence to suggest that adventitious reinforcement of untargeted behavior is a mechanism of behavior reduction in DRO treatment based on increases in untargeted behavior when a DRO is implemented (e.g., Jessel et al., 2015; Rey et al., 2020a). As such, the increase in untargeted behavior could act as a replacement for challenging behavior reduced during treatment. For example, Participant 306 in this study engaged in stable responding on the screen background which suggests that adventitious reinforcement of untargeted behavior occurred. However, untargeted behavior did not occur consistently for the other five participants in this study. If untargeted behavior occurs for sustained periods, researchers and practitioners might be interested in whether the untargeted behavior is a new or different topography of problem behavior or appropriate behavior.

Evaluations of the occurrence of untargeted behavior across contexts in a renewal paradigm may provide additional information on potential relations between untargeted 
responding and renewal of the target response. It is possible that the occurrence of untargeted behavior is related to the level of renewal (e.g., less renewal may occur if untargeted behavior is elevated in the treatment phase or the renewal test). In this study, we measured one possible topography of untargeted behavior in the form of background clicks, but it is possible that participants engaged in unmeasured topographies of untargeted behavior. Future studies could compare the occurrence or intensity of renewal following DRO while simultaneously measuring a wider set of potential topographies of untargeted behavior. In the present study, both positive and negative relations between the occurrence of renewal and untargeted behavior in the renewal test were demonstrated, but the nature of the possible association was not further explored, and future investigation of these relations is warranted.

Non-human animal and human-operant translational studies of renewal are common in the literature, but less research has assessed the prevalence of renewal in clinical settings following socially significant reduction of problem behavior (Muething et al., 2020). Studying renewal in clinical settings will provide information critical to improving clinical practice. For example, future research should evaluate the interplay between treatment integrity and renewal. Challenging behavior may relapse when treatment integrity decreases (Volkert et al., 2009) and conversely, treatment integrity may decrease when challenging behavior increases or relapses (Mitteer et al., 2018). Future research should assess how various treatment integrity levels during treatment or during a relapse test might impact renewal and other forms of relapse. In addition to studying the prevalence of renewal and the conditions under which renewal occurs in clinical settings, future research should also work to identify methods for mitigating the occurrence of renewal or decreasing the intensity or persistence of renewal of challenging behavior. For example, one possible mitigation technique is implementing treatment in multiple contexts, 
which may decrease the occurrence of renewal when context changes following treatment and contribute to generalization of treatment effects.

In clinical populations, context changes occur when alternating between therapy settings, home, and extracurricular activities. Relapse has been demonstrated following a context change from therapy to home settings (Saini et al., 2018), and recent research is increasingly investigating methods for reducing renewal in human populations (Sullivan et al., 2018). Sullivan et al. compared a traditional (i.e., sequential) model of renewal (ABA) with a nonsequential model of renewal (ACA), in which the $\mathrm{C}$ phase rapidly alternated between baseline and treatment schedules of reinforcement to more closely approximate repeated context changes during clinical treatment. For example, a client may attend outpatient treatment each weekday and return home following treatment sessions. The client is frequently alternating between treatment and home (i.e., baseline) contexts multiple times each day. Sullivan et al. found that renewal occurred at similar levels for both sequential and nonsequential renewal, suggesting that relapse during context changes is widespread and more complicated than a sequential progression from baseline to treatment and post-treatment environments and that additional research investigating when renewal occurs in human populations is imperative.

The current experiment demonstrates the occurrence of renewal following reduction of behavior using omission training according to a DRO 10-s schedule. Renewal occurred for all six participants, and for five out of six participants, renewal was transient. This study and the growing body of literature on renewal following common clinical procedures highlight that renewal is common and clinicians should be prepared for renewal when context changes after treatment. Researchers and practitioners should continue to assess when renewal occurs, under what context changes it occurs, which interventions precede its occurrence, which interventions 
yield more or less intense levels of renewal, and how to mitigate renewal following common behavior reduction treatments such as differential reinforcement. 


\section{References}

Bouton, M. E., \& Ricker, S. T. (1994). Renewal of extinguished responding in a second context. Animal Learning \& Behavior, 22(3), 317-324. https://doi.org/10.3758/BF03209840

Bouten, M. E., \& Todd, T. P. (2014). A fundamental role for context in instrumental learning and extinction. Behavioural Processes, 104, 13-19. https://doi.org/10.1016/j.beproc.2014.02.012

Bouton, M. E., Todd, T. P., Vurbic, D., \& Winterbauer, N. E. (2011). Renewal after the extinction of free-operant behavior. Learning \& Behavior, 39(1), 57-67. https://doi.org/10.3758/s13420-011-0018-6

Craig, A. R., Cunningham, P. J., Sweeney, M. M., Shahan, T. A., \& Nevin, J. A. (2018). Delivering alternative reinforcement in a distinct context reduces its counter-therapeutic effects on relapse. Journal of the Experimental Analysis of Behavior, 109(3), 492-505. https://doi.org/10.1002/jeab.431

Critchfield, T. S. (2011). Translational contributions of the experimental analysis of behavior. The Behavior Analyst, 34(1), 3-17. https://doi.org/10.1007/BF03392227

Homer, A. L. \& Peterson, L. (1980). Differential reinforcement of other behavior: A preferred response elimination procedure. Behavior Therapy, 11(4), 449-471. https://doi.org/10.1016/S0005-7894(80)80063-3

Ibanez, V. F., Piazza, C. C., \& Peterson, K. M. (2019). A translational evaluation of renewal of inappropriate mealtime behavior. Journal of Applied Behavior Analysis, 52(4), 10051020. https://doi.org/10.1002/jaba.647 
Jessel, J., Borrero, J. C., \& Becraft, J. L. (2015). Differential reinforcement of other behavior increases untargeted behavior. Journal of Applied Behavior Analysis, 48(2), 402-416. https://doi.org/10.1002/jaba.204

Jessel, J. \& Ingvarsson, E. T. (2016). Recent advances in applied research on DRO procedures. Journal of Applied Behavior Analysis, 49(4), 991-995. https://doi.org/10.1002/jaba.323

Kearns, D. N. \& Weiss, S. J. (2007). Contextual renewal of cocaine seeking in rats and its attenuation by the conditioned effects of an alternative reinforcer. Drug and Alcohol Dependence, 90, 193-202. https://doi.org/10.1016/j.drugalcdep.2007.03.006

Kelley, M. E., Liddon, C. J., Ribeiro, A., \& Greif, A. E. (2015). Basic and translational evaluation of renewal of operant responding. Journal of Applied Behavior Analysis, 48(2), 390-401. https://doi.org/10.1002/jaba.209

Kestner, K. M., Diaz-Salvat, C. C., St. Peter, C. C., \& Peterson, S. M. (2018). Assessing the repeatability of resurgence in humans: Implications for the use of within-subject designs. Journal of the Experimental Analysis of Behavior, 110(3), 545-552. https://doi.org/10.1002/jeab.477

Kimball, R. T., Greer, B. D., Randall, K. R., \& Briggs, A. M. (2020). Investigations of operant ABA renewal during differential reinforcement. Journal of the Experimental Analysis of Behavior, 113(1), 187-205. https://doi.org/10.1002/jeab.577

Lattal, K. A. \& St. Peter Pipkin, C. (2009). Resurgence of previously reinforced responding: Research and application. The Behavior Analyst Today, 10(2), 254-266. https://doi.org/10.1037/h0100669 
Lattal, K. A., Cancado, C. R.X., Cook, J. E., Kincaid, S. L., Nighbor, T. D., \& Oliver, A. C. (2017). On defining resurgence. Behavioural Processes, 141, 85-91. https://doi.org/10.1016/j.beproc.2017.04.018

Liddon, C. J., Kelley, M. E., Rey, C. N., Liggett, A. P., \& Ribeiro, A. (2018). A translational analysis of $\mathrm{ABA}$ and $\mathrm{ABC}$ renewal of operant behavior. Journal of Applied Behavior Analysis, 51(4), 819-830. https://doi.org/10.1002/jaba.496

Mitteer, D. R., Greer, B. D., Fisher, W. W., Briggs, A. M., \& Wacker, D. P. (2018). A laboratory model for evaluating relapse of undesirable caregiver behavior. Journal of the Experimental Analysis of Behavior, 110(2), 252-266. https://doi.org/10.1002/jeab.462

Muething, C., Call, N., Pavlov, A., Ringdahl, J., Gillespie, S., Clark, S., Lomas Mevers, J. (2020). Prevalence of renewal of problem behavior during context changes. Journal of Applied Behavior Analysis, 53(3), 1485-1493. https://doi.org/10.1002/jaba.672

Nakajima, S., Urushihara, K., \& Masaki, T. (2002). Renewal of operant performance formerly eliminated by omission or noncontingency training upon return to the acquisition context. Learning and Motivation, 33, 510-525. https://doi.org/10.1016/S0023-9690(02)00009-7

Nevin, J. A., Mace, F. C., DeLeon, I. G., Shahan, T. A., Shamlian, K. D., Lit, K., Sheehan, T., Frank-Crawford, M. A., Trauschke, S. L., Sweeney, M. M., Tarver, D. R., \& Craig, A. R. (2016). Effects of signaled and unsignaled alternative reinforcement on persistence and relapse in children and pigeons. Journal of the Experimental Analysis of Behavior, 106(1), 34-57. https://doi.org/10.1002/jeab.213 
Novak, M. D., Blackman, A. L., Erath, T. G., \& DiGennaro Reed, F. D. (2020). Operant renewal of desirable behavior in a simulated workplace: A translational model. Journal of the Experimental Analysis of Behavior, 113(1), 172-186. https://doi.org/10.1002/jeab.566

Poling, A. \& Ryan, C. (1982). Differential-reinforcement-of-other-behavior schedules. Behavior modification, 6(1), 3-21. https://doi.org/10.1177/01454455820061001

Romano, L. M. \& St. Peter, C. C. (2017). Omission training results in more resurgence than alternative reinforcement. Psychological Record, 67, 315-324. https://doi.org/10.1007/s40732-016-0214-Z

Saini, V. \& Mitteer, D. R. (2020). A review of investigations of operant renewal with human participants: Implications for theory and practice. Journal of the Experimental Analysis of Behavior, 113(1), 105-123. https://doi.org/10.1002/jeab.562

Saini, V., Sullivan, W. E., Baxter, E. L., DeRosa, N. M., \& Roane, H. S. (2018). Renewal during functional communication training. Journal of Applied Behavior Analysis, 51(3), 603609. https://doi.org/10.1002/jaba.471

Schindler, H. R., \& Horner, R. H. (2005). Generalized reduction of problem behavior of young children with autism: Building trans-situational interventions. American Journal on Mental Retardation, 110, 36-47. https://doi.org/10.1352/0895$\underline{8017(2005) 110<36: G R O P B O>2.0 . C O ; 2}$

Schlund, M. W., Ludlum, M., Magee, S. K., Tone, E. B., Brewer, A., Richman, D. M., \& Dymond, S. (2020). Renewal of fear and avoidance in humans to escalating threat: Implications for translational research on anxiety disorders. Journal of the Experimental Analysis of Behavior, 113(1), 153-171. https://doi.org/10.1002/jeab.565 
Sullivan, W. E. \& Roane, H. S. (2018). Incorporating choice in differential reinforcement of other behavior arrangements. American Psychological Association, 23(2), 130-137. https://doi.org/10.1037/bdb0000079

Rey, C. N., Betz, A. M., Sleiman, A. A., Kuroda, T., Podlesnik, C. A. (2020). Adventitious reinforcement during long-duration DRO exposure. Journal of Applied Behavior Analysis, 1-14. https://doi.org/10.1002/jaba.697

Rey, C. N., Thrailkill, E. A., Goldberg, K. L., \& Bouton, M. E. (2020). Relapse of operant behavior after response elimination with an extinction or an omission contingency. Journal of the Experimental Analysis of Behavior, 113(1), 124-140. https://doi.org/10.1002/jeab.568

Robinson, T. P. \& Kelley, M. E. (2020). Renewal and resurgence phenomena generalize to Amazon's Mechanical Turk. Journal of the Experimental Analysis of Behavior, 113(1), 206-213. https://doi.org/10.1002/jeab.576

Vila, J., Rojas-Iturria, F., \& Bernal-Gamboa, R. ABA renewal and spontaneous recovery of operant performance formerly eliminated by omission training. Learning and Motivation, 70, 1-10. https://doi.org/10.1016/j.lmot.2020.101631

Volkert, V. M., Lerman, D. C., Call, N. A., \& Trosclair-Lasserre, N. T. (2009). An evaluation of resurgence during treatment with functional communication training. Journal of Applied Behavior Analysis, 42(1), 145-160. https://doi.org/10.1901/jaba.2009.42-145

Wacker, D. P., Harding, J. W., Berg, W. K., Lee, J. F., Schieltz, K. M., Padilla, Y. C., Nevin, J., \& Shahan, T. A. (2011). An evaluation of persistence of treatment effects during long- 
term treatment of destructive behavior. Journal of the Experimental Analysis of Behavior, 96(2), 261-282. https://doi.org/10.1901/jeab.2011.96-261

Wathen, S. N. \& Podlesnik, C. A. (2018). Laboratory models of treatment relapse and mitigation techniques. Behavior Analysis: Research and Practice, 18(4), 362-387.

https://doi.org/10.1037/bar0000119 
Figure 1
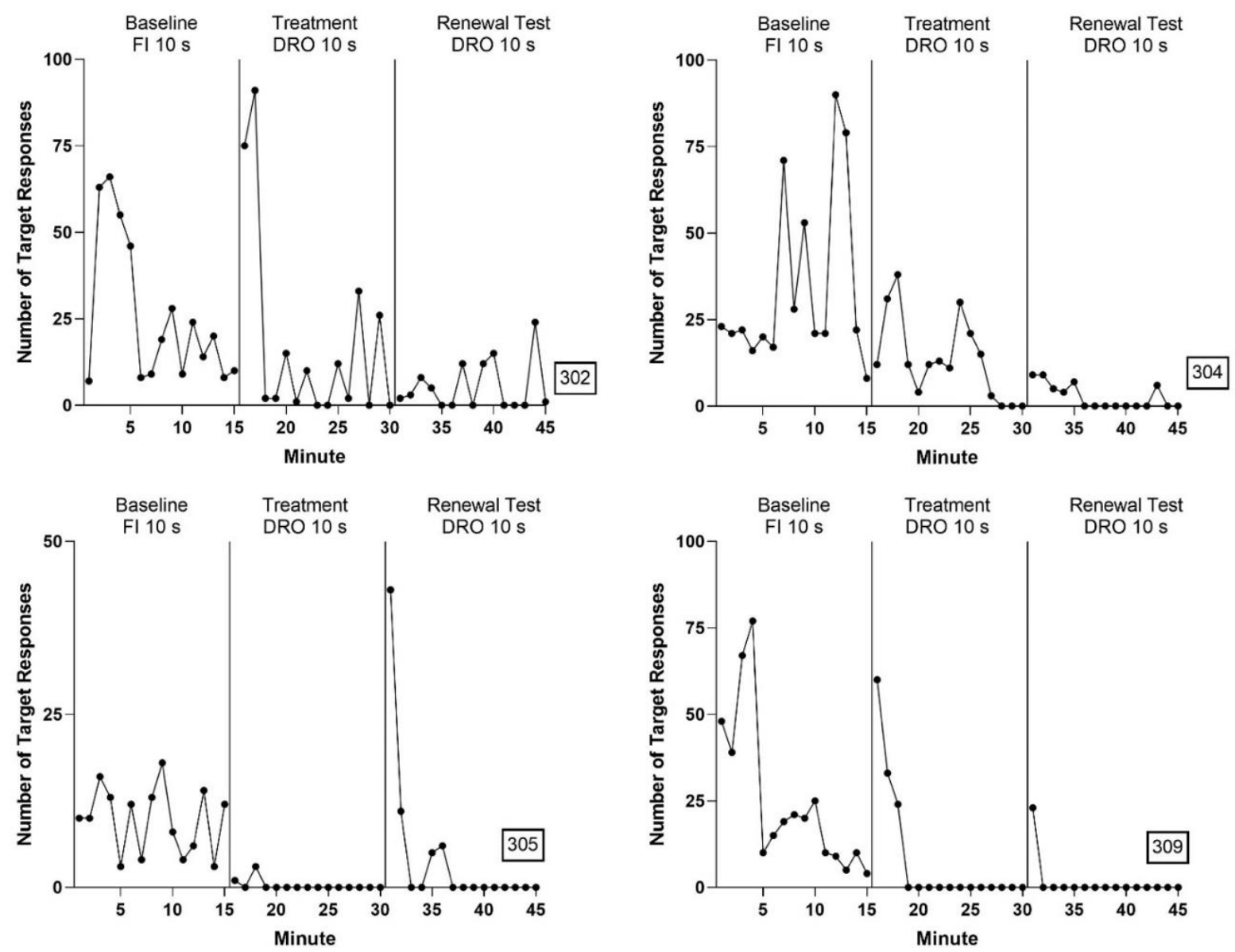

Note. The number of target responses in each min of the baseline, DRO treatment, and renewal test phases for all excluded participants. Please note that the y-axis scale varies across graphs based on participant responding. 
Figure 2
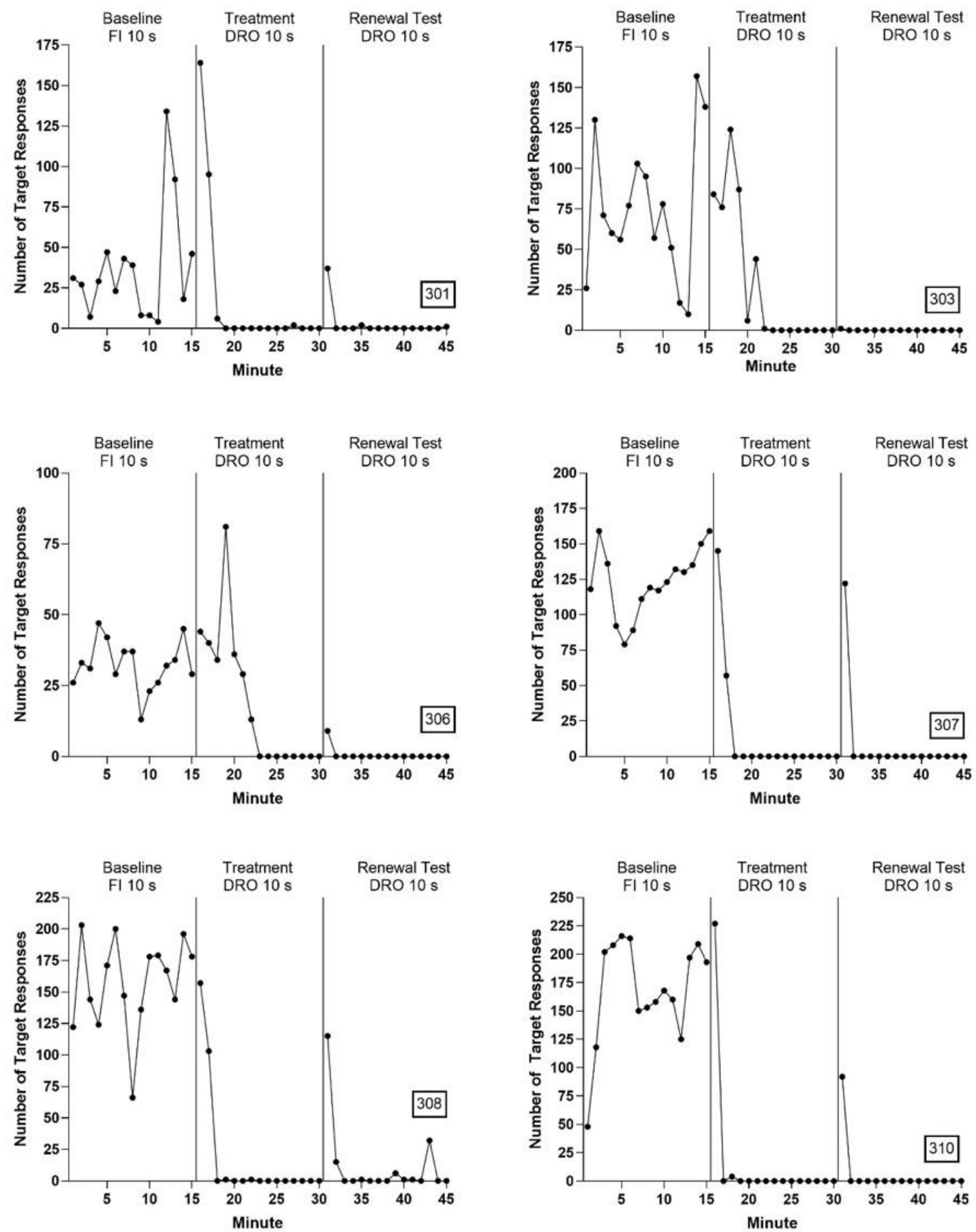

Note. Number of target responses in each min of baseline, DRO treatment, and renewal test phases for all participants included in data analysis. Please note the variable y-axis scales across graphs. 
Figure 3
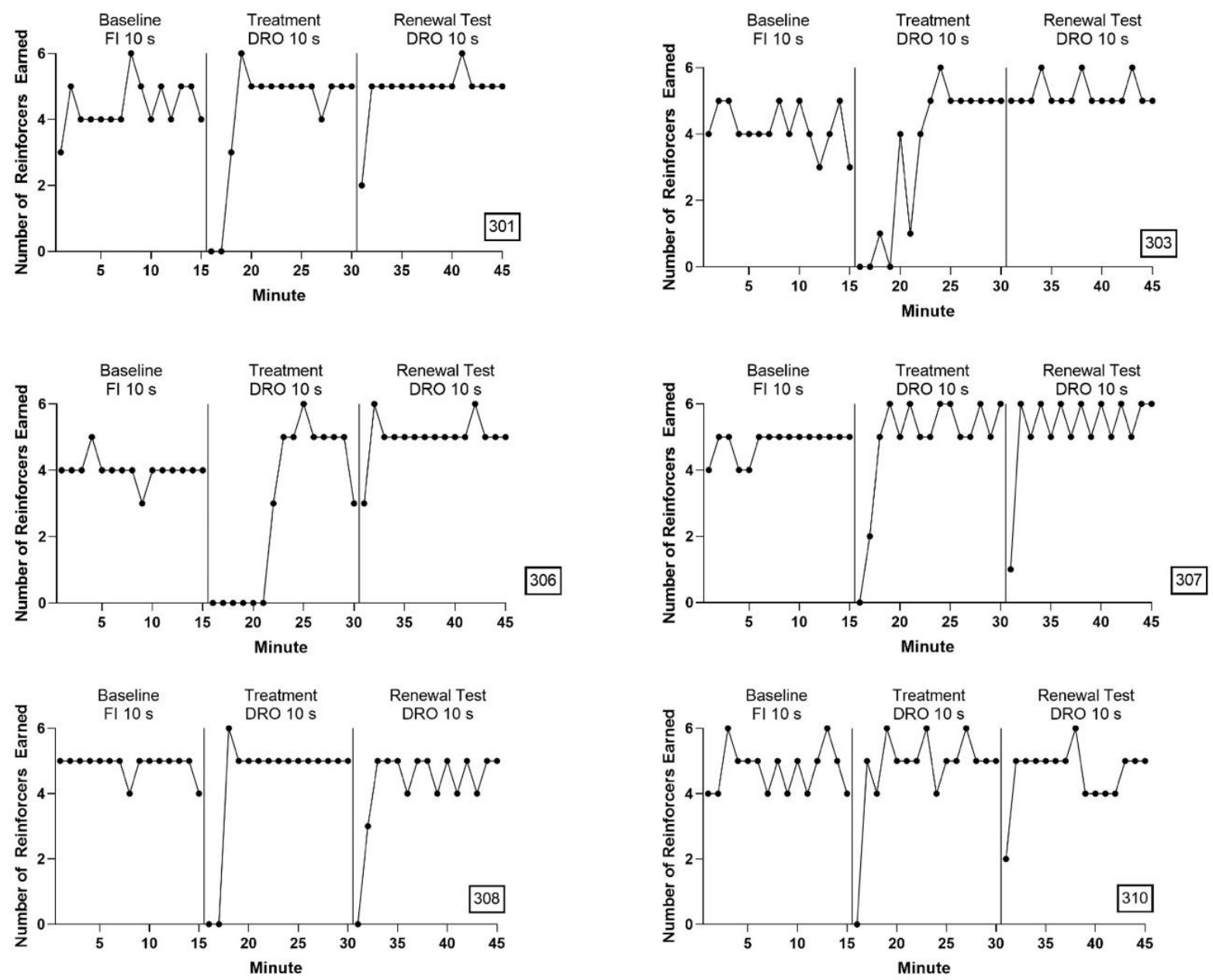

Note. The number of reinforcers delivered in each min of the baseline, DRO, and renewal test phases for all included participants. 


\section{Figure 4}
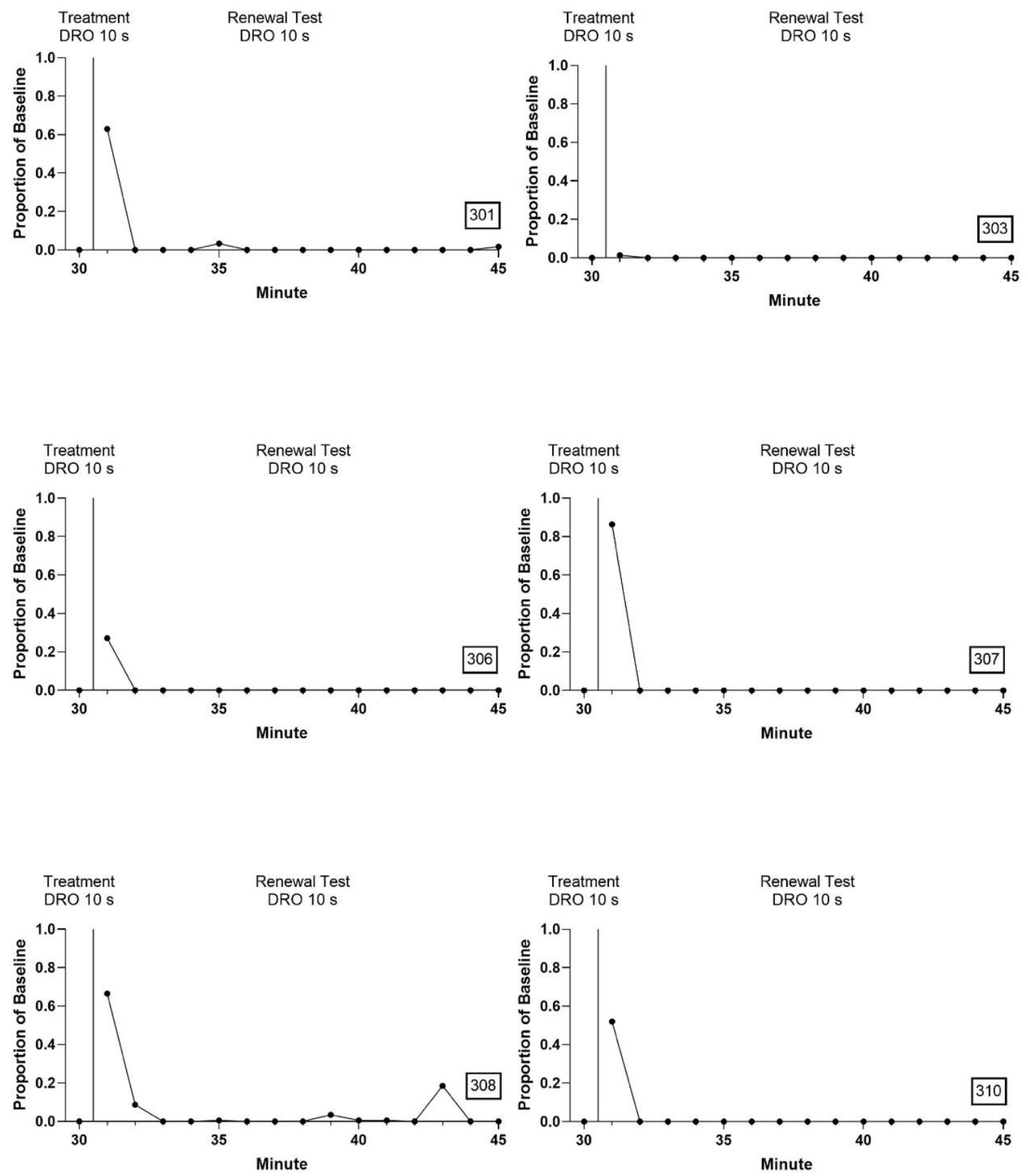

Note. The proportion of baseline for the last min of the DRO treatment phase and all $15 \mathrm{~min}$ of the renewal test phase for all participants included in data analysis. 
Figure 5
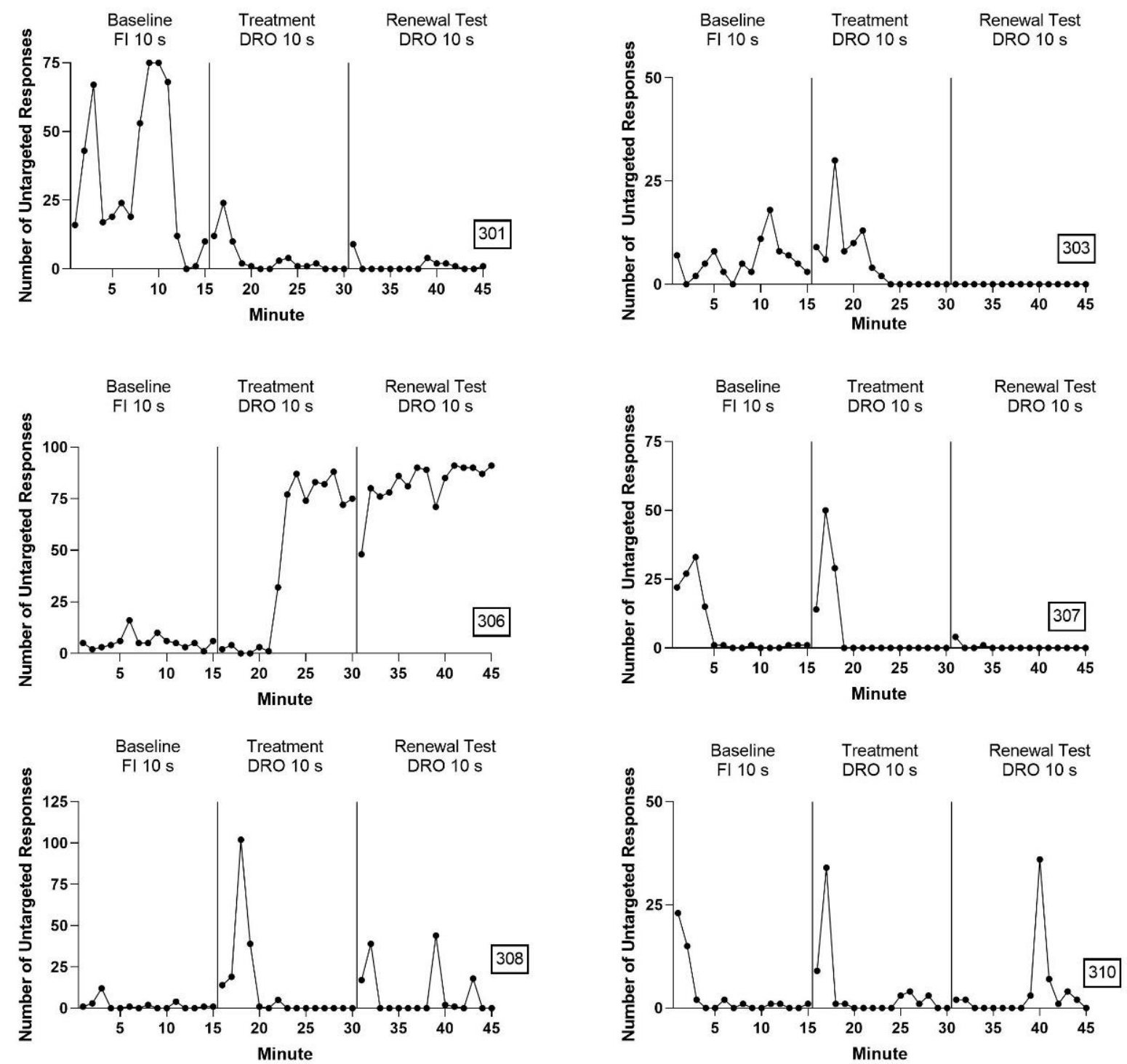

Note. Number of untargeted responses in each min of the baseline, DRO treatment, and renewal test phases for all included datasets. Please note that the y-axis differs across graphs based on variable rates of participant responding. 
Table 1

Consummatory Response Time

\begin{tabular}{cccccc}
\hline Participant & $\begin{array}{c}\text { Included or } \\
\text { Excluded }\end{array}$ & $\begin{array}{c}\text { Time with CM } \\
\text { in Phase 1 }\end{array}$ & $\begin{array}{c}\text { Time with CM } \\
\text { in Phase 2 }\end{array}$ & $\begin{array}{c}\text { Time with CM } \\
\text { in Phase 3 }\end{array}$ & $\begin{array}{c}\text { Total Time } \\
\text { with CM }\end{array}$ \\
\hline 301 & Included & 1.87 & 2.04 & 1.90 & 5.81 \\
302 & Excluded & 1.27 & 1.60 & 2.58 & 5.45 \\
303 & Included & 1.34 & 0.56 & 1.87 & 3.77 \\
304 & Excluded & 2.51 & 2.19 & 2.46 & 7.16 \\
305 & Excluded & 1.20 & 1.64 & 1.75 & 4.59 \\
306 & Included & 1.46 & 1.52 & 2.19 & 5.17 \\
307 & Included & 1.38 & 1.21 & 1.13 & 3.72 \\
308 & Included & 1.61 & 2.17 & 2.17 & 5.95 \\
309 & Excluded & 1.43 & 1.18 & 1.38 & 3.99 \\
310 & Included & 2.08 & 2.00 & 2.73 & 6.81 \\
\hline
\end{tabular}

Note. The time spent with the consummatory response present and participants engaging in the consummatory response (i.e., clicking the "collect point" message) for each phase and the total time for the whole session. 


\section{Appendix A}

\section{Questionnaire}

For research staff:

Participant ID:

Date: Time:

RA name:

Instructions: The purpose of the following questions is to collect information about your demographic variables and your impressions of the study. Please skip any questions that do not feel comfortable answering.

What is your age in years?

- I prefer to not answer

How do you currently describe your gender identity?

- I prefer to not answer

How do you identify your racial and ethnic background? Please select all that apply to you:

- American Indian or Alaska Native

- Asian 
- Black or African American

- Hispanic, Latinx/Latine, or Spanish Origin

- Middle Eastern or North African

- Native Hawaiian or Other Pacific Islander

- White

- Other/prefer to self-describe:

- Unknown

- I prefer to not answer

Do you have (or suspect that you have) a color vision deficiency? If yes, what form?

Please describe what happened and what you did during the session(s).

Please describe any strategies that you may have used to earn points. Did any of your strategies change across the experiment?

What do you think we are trying to learn from this study? 


\section{Appendix B}
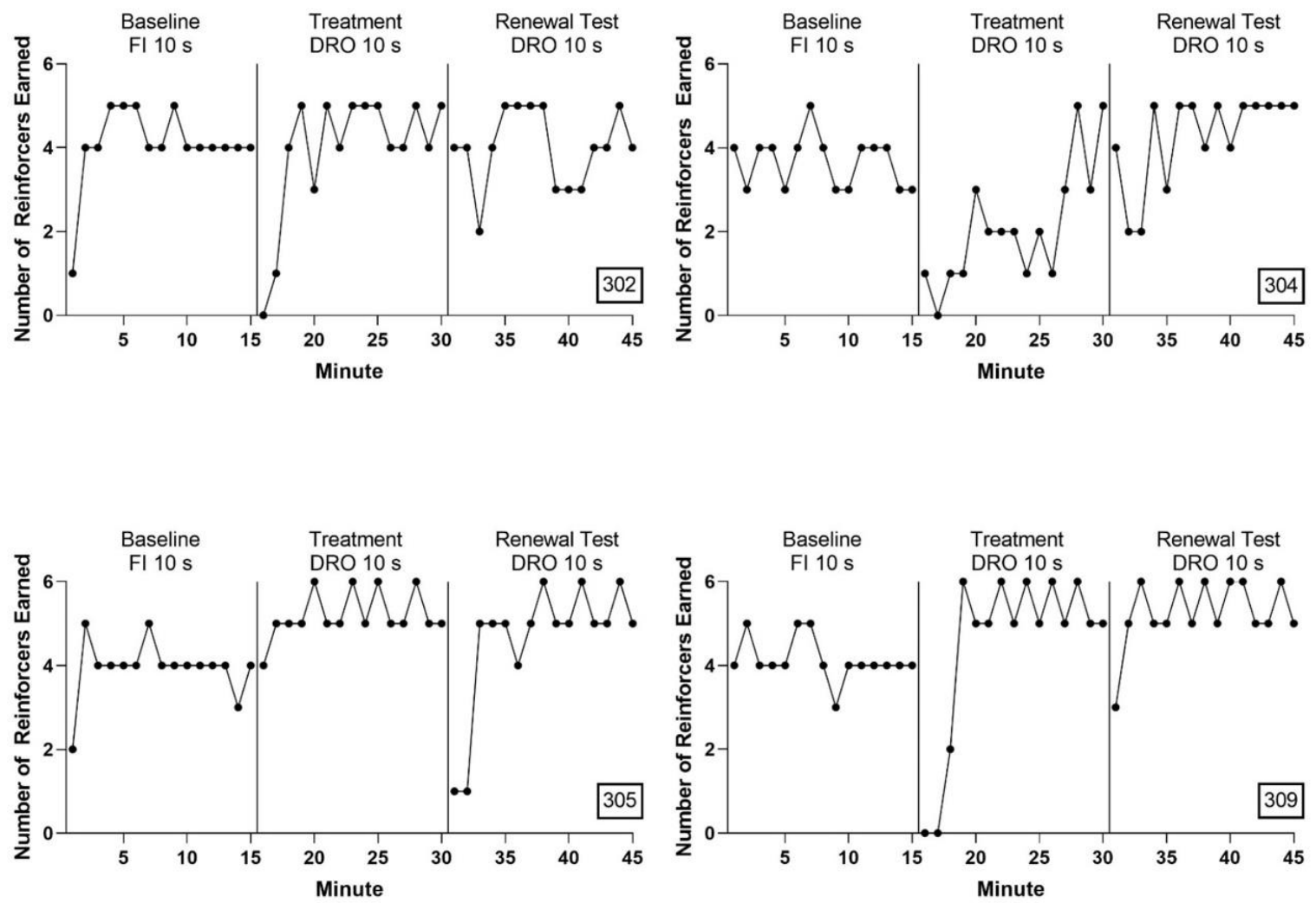

Note. The number of reinforcers delivered in each min of the baseline, DRO treatment, and renewal test phases for all excluded participants. 

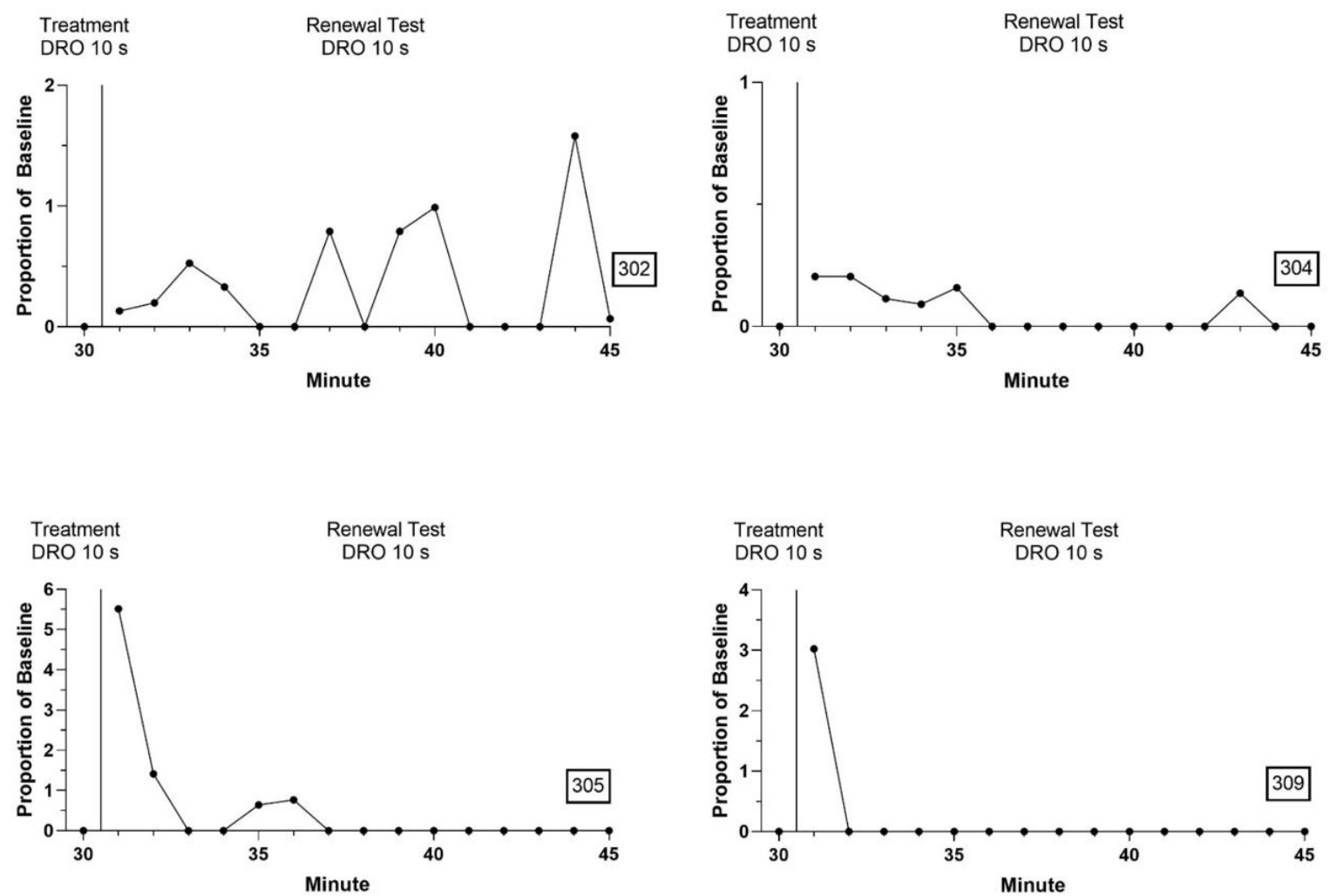

Note. The proportion of baseline for the last min of treatment and the $15 \mathrm{~min}$ of the renewal test for all excluded participants. Please note that the y-axis scale varies across graphs. 